\title{
Stable Coalition Structures with Fixed Decision Scheme*
}

\author{
Guillaume Haeringer \\ This version: 7 th July 2000
}

${ }^{*}$ Comments and criticisms from Carmen Beviá, Pierre Dehez, Carmen Herrero, Michel Le Breton, Jordi Massó, Jean-François Mertens, Thomas Palfrey, Ulrich Schmidt, Gisèle Umbhauer, seminar audiences at CORE and "Ecole de Printemps" of CORE-GREQAM in Aix-en-Provence, and particularly Hideo Konishi for comments and suggestions that greatly improved the exposition of this work are gratefuly acknowledged. Part of this work was done while I was visiting the Institute of Mathematical Economics at Bielefeld University under the European research programme "Game-theoretic approaches to cooperation and exchange of information, with economic applications." Financial support from the European Commission, contract number HPMF-CT-1999-00284, is gratefuly acknowledged. All remaining errors are mine. 


\begin{abstract}
This paper studies the stability of a finite local public goods economy in horizontal differentiation, where a jurisdiction's choice of the public good is given by an exogenous decision scheme. In this paper, we characterize the class of decision schemes that ensure the existence of an equilibrium with free mobility (that we call Tiebout equilibrium) for monotone distribution of players. This class contains all the decision schemes that lie between the Rawlsian decision scheme and the median voter with mid-distance of the two median voters when there are ties. In the last part of the paper, we prove the non-emptiness of the core of this coalition formation game.
\end{abstract}

Keywords: Coalition structures, Tiebout equilibrium, $\mathcal{C}$-stability, decision scheme.

Journal of Economic Literature Classification: C71, D70, H73.

Guillaume Haeringer

Departament d'Economia i d'Història Econòmica and CODE

Universitat Autònoma de Barcelona

08193 Bellaterra, Spain

Tel: +34935812505 Fax: +34935812461

E-mail: ghaeringer@volcano.uab.es 


\section{Introduction}

The aim of this paper is to study the stability of coalition structures, in non-cooperative and cooperative frameworks. We advocate that coalitions result from a contest between two opposite forces. On the one hand, individuals might be attracted by big communities, clubs or networks, e.g., when the power of coalitions increases with its size - as suggested by Demange (1994) — or when there are increasing returns to scale. On the other hand, individuals may feel better off in small structures, and seek to join small groups rather than big ones. For instance, in small groups agents are usually more likely to be part of some kind of decision making processes than in bigger ones. If one of these two forces has no or little influence, trivial equilibria may be reached. ${ }^{1}$ By contrast, when both forces have comparable effects, the outcome of the coalition formation process becomes harder to predict, and finding stable coalition structures is more intricate.

Economic analysis offers a wide variety of problems that can be reformatted in terms of coalition formation, that range from party formation in political economy to the origin of cartels in industrial organization. In all these situations, a starting point is that individuals carry out economic or political activities as coalitions, which are often determined endogenously. Local public good economies are one of the most appealing frameworks for the study of coalition structures. Such an economy is defined as follows. There is a set of potential jurisdictions, each of them supplying a public good bundle. Public goods have the property that the same amount of the good is supplied to all agents. Local public goods, as introduced by Tiebout (1956), are public goods whose consumption is only possible for agents living in the jurisdiction that provides them. In models $\grave{a}$ la Tiebout, each jurisdiction has to finance the production of the public goods bundle it supplies. The two forces that have been mentioned above are clearly defined in this context. On the one hand, agents seek to join the jurisdiction that provides their most preferred public good bundle; so, if each jurisdiction chooses its own public good with respect to the tastes of its inhabitants, it is obvious that agents may favor small communities, where their tastes are likely to have a large impact on the choice of the public good. On the other hand, when the cost of the public good production is independent of the number of users (no crowding effects), the tax level paid by an agent decreases with

\footnotetext{
${ }^{1}$ In this paper, "equilibrium" means a state such that no player migrates from a coalition to another one.
} 
the number of agents in the jurisdictions (or agents' wealth), and therefore agents also care about the 'size' of the jurisdictions they may enter. Consequently they may favor those with many members rather than those that supply their preferred public good bundle.

In this paper, we propose a coalition formation model in the vein of local public goods economies, with the following characteristics. Individuals in a finite population have to choose the location of a public good/facility. Agents may form jurisdictions, and each jurisdiction finances its own public goods. We suppose that a player's utility in a coalition is increasing with the number of agents in the coalition to which she belongs, and decreasing with the distance between the agent's location and that of her coalition's public good. A major assumption that we make is the existence of a decision scheme, which refers to as a collective choice within each jurisdiction. The rationale for a decision scheme is the following. In each jurisdiction, the public good supplied is common to all inhabitants, and as agents may have different tastes, it is reasonable to think that the choice of the public good is made through some decision process, i.e., a decision scheme. For instance, this latter can be either the result of a vote, or the decision of a social planner. Our purpose is to study the impact of this assumption on the existence of stable coalition structures, i.e., states in which no player move from one jurisdiction to another, and to identify which decision schemes ensure the existence of stable coalition structures.

In economies with a continuum of agents, several authors imposed a fixed decision scheme. Eppel, Filimon, and Romer (1984, 1993), Konishi (1996), Jehiel and Scotchmer (1997) and Alesina and Spolaore (1997) supposed that consumers vote, but these latters also used a social planer who maximizes the sum of individual utilities. However, in previous models with a finite number of agents, the existence of a decision scheme was not assumed. The model we propose in this paper contrasts with the existing literature in that respect. $^{2}$

\footnotetext{
${ }^{2}$ One finds in the literature two models of a finite local public good economy with a fixed decision scheme, Rose-Ackerman (1979) and Greenberg and Shitovitz (1988). These two models supposed that the decision scheme is a majority vote. Hence, the choice of the jurisdiction corresponds to the choice of the median voter - see Black (1948). Greenberg and Shitovitz (1988) studied a local public good economy with a finite set of agents and a fixed decision scheme - the majority vote. However, their concern is about the existence of a market equilibrium, and not about agents' migrations. Rose-Ackerman (1979) studied a model where consumers are allowed to migrate, but she also assumed that there is a market for land. Her main concern was about the equilibria of the economy, when the land market clears. However, her model differs from ours in that we do not fix an exogenous
} 
A major consequence of a fixed decision scheme is the following. In models that do not assume the existence of decision scheme, if a consumer joins a jurisdiction the choice of the public good supplied remains unchanged. Yet, in our model, this is not the case. This induces on individual moves both positive and negative externalities. We show that the net effects of these externalities are hard to disentangle. Indeed, when an agent enters a jurisdiction, she may shift the location of the public good, which in turn may affect the payoffs of those individuals who already belong to that jurisdiction - and individuals' payoffs in the former jurisdiction as well. In our model, unlike in Greenberg and Weber's (1993), or in Konishi, Le Breton, and Weber's (1998), the decision of the jurisdiction can be affected by a migrant. This implies that the migrant is not always welcome. A major consequence is that different equilibrium concepts may not coincide, which is the case in in Greenberg and Weber's (1986) model. This impact of the decision scheme is not shared with models with a continuum of agents. In these economies, each individual has a zero weight in the population. If an agent enters a jurisdiction, she has no influence on the decision scheme. ${ }^{3}$ For instance, Westhoff (1977, p. 86) assumed that "each individual takes the levels of the public good and tax rate offered by his and other communities as fixed, that is, he does not account for the effect of consumer migration."

Another consequence of the decision scheme assumption is that the local public good economy model can be understood as a two-stage game. The first stage is devoted to the formation of coalitions, and the second stage is devoted to the choice of the public good. This allows us to define an associated hedonic game, that has proved useful for the study of coalition formation. Hedonic games are games in which the utility of a player depends on the identity of other members of her coalition. These games were first defined by Drèze and Greenberg (1980), and recently developed by Banerjee, Konishi, and Sönmez (1998), and Bogomolnaia and Jackson (1998).

An interesting feature of our model is about the differentiation of public goods. In the local public good economy literature, most authors focused on the vertical differentiation case. Public good bundles proposed in different jurisdictions differ in quality, in the level supplied, or in both. However,

number of jurisdictions.

${ }^{3}$ Jehiel and Scotchmer (1997) bypassed this by adding a constraint on consumers' moves. They assumed that only coalitions with a size greater than a minimal threshold are authorized to move. Thus, moves in their model have the same properties as in ours. However, Jehiel and Scotchmer could not make anymore the difference between individual and collective moves. 
little attention has been paid to the horizontal case, when jurisdictions have to choose the location of the public good. This difference is important since in the vertical case, if the tax rate were to remain unchanged, all consumers would agree to choose the highest level/quality of public good. In the horizontal case, the cost of the public good is fixed and a change in the location of the public good yields some players better off and other worse off. Hence, our model follows the same line of research as Alesina and Spolaore's (1997), Jehiel and Scotchmer's (1997), or more rencently Le Breton and Weber's (2000), who studied models where agents have to choose the location of a government. Unlike us, their models assume the existence of a continuum of agents. As we shall see, our results sharply contrast with theirs.

We consider two frameworks, non-cooperative and cooperative, and study the main equilibrium concepts to which they are usually associated, namely Tiebout-stability and $\mathcal{C}$-stability respectively. Depending on whether individuals are free or not to leave and to enter a jurisdiction, and on whether or not collective moves are allowed, different concepts of equilibrium can be obtained - Nash, Strong-Nash, Coalition-Proof or Core like concepts are the most common ones. In other words, a local public good economy can be modeled either as a non-cooperative game - e.g., Westhoff (1977) or Konishi, Le Breton, and Weber (1998) — or as a cooperative game - Guesnerie and Oddou (1979). This paper will embody these two frameworks. The two concepts of stability that we shall study are that of Tiebout-stability and $\mathcal{C}$-stability. In the former, individuals are free to leave and to enter any coalition, without the consentment of the other players, but only individual decisions are allowed. In the latter, group decisions are allowed and the free exit assumption still holds. However, when entering a coalition, a group of agents must have the consentment of all players in that coalition. We also assume that with these two stability concepts, players are always free to create a new coalition. In other words, the maximal number of coalitions is not fixed.

It turns out that when the distribution of players in the space is monotone, a Tiebout equilibrium always exists if and only if the decision scheme is between the Ralwsian decision scheme and the median voter, if the tiebreaking rule consists of taking the mid-point between the two median agents when the number of players is even in the coalition. As we make the assumption that players have the same utility functions, the Rawlsian decision scheme corresponds to the mid-point between the most "marginalistic" agents of a jurisdiction, i.e., the pair of agents with the greatest distance 
between their respective location. It can be observed that this latter coincides with the median voter only when the distribution of agents on the characteristic space is uniform.

The paper is organized as follows. The next section presents the local public good economy model that we use throughout. It contains a description of the agents, their preferences, and the main assumptions we make on decision schemes. In section 3, we study equilibria with free mobility, that we call Tiebout equilibria, and characterize the decision schemes that ensure the existence of a Tiebout equilibrium. In section 4 we study the case in which multilateral moves are allowed, but without the free entry assumption. These equilibria are called $\mathcal{C}$-stable coalition structures. In section 5 , we study the intersection of the Tiebout equilibria and $\mathcal{C}$-stable coalition structures. We show that a Tiebout-stable coalition structure may not be $\mathcal{C}$-stable and vice-versa, yet they can have a non-empty intersection. This contrasts with Greenberg and Weber (1986) who showed that with a model similar to ours, any $\mathcal{C}$-stable coalition structure is also Tiebout-stable. We conclude in section 6. All proofs are relegated to the Appendix.

\section{The Model}

We denote by $N$ the set of agents, and assume that it is finite, $N=$ $\{1, \ldots, n\}$. A coalition $S$ is any subset of $N$, and $|S|$ denotes its size. A coalition structure $\mathscr{B}=\left\{S_{1}, \ldots, S_{k}\right\}$ is any partition of $N$ in coalitions,

$$
S_{h} \cap S_{h^{\prime}}=\varnothing, \forall h \neq h^{\prime} \text {, and } \bigcup_{h=1}^{k} S_{h}=N .
$$

For each coalition structure $\mathscr{B}$ we designate by $S(i, \mathscr{B})$ the coalition $i$ belongs to. When no confusion is possible, we write $S(i)$ instead of $S(i, \mathscr{B})$. Throughout the paper, $\subset$ and $\subseteq$ will respectively denote the weak and the strong set inclusion.

\subsection{Locations}

We suppose that agents are scattered over a subset of the real the line, $\Omega$. Each $i$ player's location is given by $p_{i} \in \Omega$. In the sequel, we will refer to $p_{i}$ as the position of agent $i$. We understand $p_{i}$ as a fundamental characteristic of player $i$, and as such $p_{i}$ does not depend on the coalition $i$ belongs to. 
A natural assumption is that $p_{i}$ is the public good location that $i$ would have chosen if she was alone. A logical consequence is that if agent $i$ has to choose between two public good locations, she will prefer the one that is closest to her ideal choice, $p_{i}$. Hence, players' preferences over locations are single peaked. However, we shall assume later that agents' utilities also depend on the coalition's size to which they belong, and thus the single peakedness property will no longer hold.

In the sequel, we will put the emphasis on the class of monotone distributions of players' positions over $\Omega$.

Definition 1 The distribution of players $\left(p_{1}, \ldots, p_{n}\right)$ is monotone if either,

$$
\text { or } \quad \mid \begin{aligned}
\left|p_{i}-p_{i-1}\right| & \geq\left|p_{i+1}-p_{i}\right|, \forall i=\{2, \ldots, n-1\}, \\
\left|p_{i}-p_{i-1}\right| & \leq\left|p_{i+1}-p_{i}\right|, \forall i=\{2, \ldots, n-1\} .
\end{aligned}
$$

We say that the distribution is strictly monotone if it is monotone and a strict inequality holds for at least one $i=\{2, \ldots, n-1\}$. The distribution is uniform if the equality holds for all $i=\{2, \ldots, n-1\}$.

In other words, in a monotone distribution, the distance between two consecutive players is always non-decreasing (or non-increasing) along $\Omega$.

We say that a coalition is connected if, when considering two players in it, all players with intermediate positions belong to the same coalition.

Definition 2 A coalition $S$ is connected if $i, k \in S$ implies that $j \in S$ for all $j$ such that $p_{i} \leq p_{j} \leq p_{k}$.

Connectedness for a coalition structure occurs provided the previous property holds across all coalitions.

Definition 3 A coalition structure $\mathscr{B}$ is connected if all coalitions $S \in \mathscr{B}$ are connected.

\subsection{Decision schemes}

The second fundamental assumption of our model is that no agent can decide by herself the public good that will be chosen in her community, unless she is the only member of that community. Thus, when several agents form a community, a unique public good will be chosen by a decision scheme. We shall assume that the decision scheme solely depends upon the positions of players. 
Definition 4 A decision scheme $F$ is an application that maps for each coalition $S$ the vector of players' positions, $\left(p_{i}\right)_{i \in S}$, to a point in $\Omega$,

$$
F: \bigcup_{S \in 2^{N} \backslash\{\varnothing\}} \Omega^{|S|} \rightarrow \Omega
$$

For each community $S, F\left(\left(p_{i}\right)_{i \in S}\right)$ will give the location of a public good in $\Omega$ that will be the public good supplied to all agents in $S$. To simplify the notation we write $F(S)$ instead of $F\left(\left(p_{i}\right)_{i \in S}\right)$. In other words, a decision scheme assigns to any coalition $S$ a point in $\Omega$, given the positions of players in $S$. We assume that $F(S)$ does not depend on agents outside $S$. We shall impose some consistency properties on the decision scheme. That is, we restrict ourselves on decision schemes having the following properties.

Assumption 1 (Pareto optimality) For any coalition $S, F(S)$ is higher or equal to the lowest position in $S$, and lower or equal to the highest position in $S$, that is,

$$
F(S) \in\left[\min _{i \in S} p_{i} ; \max _{i \in S} p_{i}\right]
$$

Pareto optimality has a simple interpretation. Any change of the value of $F(S)$ makes at least one player in $S$ worse off. Let us suppose that $F$ does not satisfy this assumption, and consider $F(S)<p_{i}=\min _{h \in S} p_{h}$. Then, taking $p_{i}$ instead of $F(S)$ makes all the members of $S$ strictly better off.

Assumption 2 (Invariance) Let $g: \mathbb{R} \rightarrow \mathbb{R}$ be any monotone increasing function and $\mu \in \mathbb{R}$. Define $p_{i}^{\prime}:=g\left(p_{i}\right)+\mu$. Then for all $i \in S \subseteq N$,

$$
\begin{array}{ll}
F\left(\left(p_{j}^{\prime}\right)_{j \in S}\right) \leq g\left(F\left(\left(p_{j}\right)_{j \in S}\right)\right)+\mu & \text { if } f \text { is convex, } \\
F\left(\left(p_{j}^{\prime}\right)_{j \in S}\right) \geq g\left(F\left(\left(p_{j}\right)_{j \in S}\right)\right)+\mu & \text { if } f \text { is concave. }
\end{array}
$$

This assumption merely says that the relative positions to the outcome of the decision scheme should not depend on the scale choosen for $\Omega$, nor its origin.

Several decision schemes satisfy these properties. The most common ones are the median voter, ${ }^{4}$ which we denote $F^{\text {med }}$, and the social planner maximizing the sum of individual utilities. Given the symmetry assumption, this latter is equivalent to minimizing the distance between each agent's

\footnotetext{
${ }^{4}$ The median voter in $S$ is the player $i$ such that the sets $\left\{j \in S: p_{j} \leq p_{i}\right\}$ and $\left\{j \in S: p_{j} \geq p_{i}\right\}$ have the same cardinality.
} 
position and the outcome of the decision scheme, which yields the mean of the positions.

Another decision scheme that satisfies theses properties is the mean of the extremes. ${ }^{5}$ It consists of taking the point at the mid-distance between the two players with the lowest and highest positions. Formally, it is given by

$$
F^{e x t}(S)=\frac{p_{\min (S)}+p_{\max (S)}}{2}
$$

where

$$
\begin{aligned}
& p_{\min (S)}=\left\{p_{i}: p_{i} \leq p_{j}, \forall j \in S\right\}, \\
& p_{\max (S)}=\left\{p_{i}: p_{i} \geq p_{j}, \forall j \in S\right\} .
\end{aligned}
$$

\subsection{Utility functions}

Each agent's utility function depends on two arguments, the distance between the choice of the coalition she belongs to and her position, and the size of her coalition. Thus, we implicitly assume that the utility of an agent does not depend on the coalitions to which she does not belong.

First, each agent $i$ in $S$ measures the difference between her position $p_{i}$ and the outcome of the decision scheme for her coalition, $F(S)$. We denote this difference by $\delta_{i}(S)$, and it is given by,

$$
\delta_{i}(S)=\left|F(S)-p_{i}\right|, \forall i \in S .
$$

We define the utility of agent $i$ as a mapping $u_{i}: \mathbb{N} \times \mathbb{R}_{+} \rightarrow \mathbb{R}$, with,

$$
u_{i}\left(|S|, \delta_{i}(S)\right)
$$

and $u_{i}$ is increasing with respect to its first argument and decreasing with respect to its second argument. Moreover, we also suppose that $u$ is concave with respect to coalition size and distance.

\footnotetext{
${ }^{5}$ Notice that Westhoff (1977), Konishi (1996), Alesina and Spolaore (1997), and Jehiel and Scotchmer (1997) all made the assumption of a uniform distribution of agents. Thus, all their results still hold when their decision scheme is replaced by the mean of the extremes. Alesina and Spolaore (1997, section VI, p. 1044) weakened the uniform distribution assumption, but they only presented how the model would change - such as the outcome of the decision scheme.
} 


\section{Assumption 3 (Concavity)}

(i) with respect to the coalitions size: For all $s \in \mathbb{N}$ and $\delta \in \mathbb{R}_{+}$,

$$
u_{i}(s+1, \delta)-u_{i}(s, \delta) \geq u_{i}(s+2, \delta)-u_{i}(s+1, \delta) .
$$

(ii) with respect to the distance $\delta$ : For all $s \in \mathbb{N}$ and $\delta, \delta^{\prime} \in \mathbb{R}_{+}$, such that $\delta \leq \delta^{\prime}$,

$$
u_{i}(s, \delta)-u_{i}(s, \delta+\varepsilon) \leq u_{i}\left(s, \delta^{\prime}\right)-u_{i}\left(s, \delta^{\prime}+\varepsilon\right), \forall \varepsilon>0 .
$$

The concavity with respect to the first argument means that the positive effect of an increase in the coalition size is greater for small coalitions than for big ones. Concavity with respect to the second argument of $u_{i}$ reads as follows. We know that if $\delta$ increases, ceteris paribus, then the utility decreases. Concavity says that for a same positive variation on $\delta$, the greater $\delta$ is, the greater the loss of utility is.

The concavity assumptions can be reinterpreted as follows. It is usually assumed that public goods are produced using private goods. The main idea behind is that of decreasing returns to scale in the production of the public good. In the model, we assume that the cost of the public good is the same for all coalition, whatever their size. This refers to as pure public goods in the terminology of Bewley (1981). ${ }^{6}$ A natural assumption is then that per capita cost of the public good decreases as population size increases, which can be understood as a weak congestion effect. In our model, we make players' preferences dependent on the difference between their position and the outcome of the decision scheme in their coalition. As we explained, our model is better understood as a local public good economy with horizontal differentiation, which is often interpreted as a location problem - see for instance Ireland (1987). Thus, in our model, it can be understood that agents experience a transportation cost, and the concavity assumption simply means that this cost is convex, such as a quadratic cost.

We also suppose that agents' utilities have the same functional form. Thus, agents only differ in their positions on $\Omega$.

\footnotetext{
${ }^{6}$ Bewley (1981) distinguished initially between two cases of public goods, pure public goods and pure public services. In the former, the cost is independent of population and in the second the cost of the public good is proportional to population. He also pointed out a third case, when the per capita cost of public goods is a $U$-shape function of the population.
} 
Assumption 4 (Symmetry) There exists a $u: \mathbb{N} \times \mathbb{R}_{+} \rightarrow \mathbb{R}$ such that

$$
u_{i}(x, y)=u(x, y), \forall x, y \in \mathbb{R}^{+}, \forall i \in N .
$$

This last assumption undoubtedly puts strong restrictions on players' preferences. It should be noted, however, that a similar assumption was already made in the literature on coalition formation. For instance, Greenberg and Weber (1986) supposed that all players have the same utility function and only differ in their income. ${ }^{7}$ However, it turns out that this assumption is crucial for the existence of a Tiebout equilibrium (see example 2 on page 18 ) or a $\mathcal{C}$-stable coalition structure (see example 4 on page 20 ).

However, this assumption implies a nice property on the mean of the extremes. This decision scheme is the unique Rawlsian decision scheme. Indeed, in any coalition $S$, the player with the lowest utility is player $i^{*}$ such that $\delta_{i}(S) \leq \delta_{j}(S), \forall j \in S$. Clearly, if the outcome of the decision scheme is not the mid-point between the two extreme players, then one can increase the utiliy of player $i^{*}$ by moving the outcome to $F^{e x t}(S)$, and if the outcome is located beyond $F^{e x t}(S)$, then there is one player with a lower utiliy than $i^{*}$.

At last, it can be observed that contrary to Greenberg and Weber (1986), Alesina and Spolaore (1997), Jehiel and Scotchmer (1997), and Konishi, Le Breton, and Weber (1998) we do not make any assumption on the functional form of $u$, such as quasi-linearity, separability, or continuity. ${ }^{8}$

\subsection{The coalition formation game}

Our model can be presented as a hedonic coalition formation game. The concept of hedonic game was first introduced by Drèze and Greenberg (1980). They defined hedonic games as those in which the utility of a player has two arguments, her consumption bundle and the coalition to which she belongs. The dependence of the utility on the members of the coalition was called by Drèze and Greenberg (1980) the "hedonic aspect". Clearly, the model we present has this feature too, since the utility of an agent depends on the choice of the coalition (the public good location in our case), and also on the identity of the members of the coalition through its size.

\footnotetext{
${ }^{7}$ A similar assumption is found in other models, such as in Eppel, Filimon, and Romer (1984, 1993), Jehiel and Scotchmer (1997), and Alesina and Spolaore (1997).

${ }^{8}$ Most of the utility functions that we shall use in the examples or in the proofs are not continuous. However, it is easy to see that these utility functions can be extended in order to be continuous. Continuity of these utility functions would simply have rendered the examples or the proofs more cumbersome.
} 
Bogomolnaia and Jackson (1998), Banerjee, Konishi, and Sönmez (1998), and Barberà and Gerber (1999) have recently refined Drèze and Greenberg's (1980) model in more tractable ways. They focused on "pure hedonic games", i.e., hedonic games in which no redistributional issue has to be solved within coalitions. In these games, a player's payoff only depends on the composition of members of the coalition she belongs to. Our model can also be seen as a pure hedonic game, since the decision scheme $F$ is fixed for all coalitions and known by all players. We suppose that information is perfect and that players are rational. Thus, each player is able to compute the utility she will obtain for any coalition she may belong to. This allows us to define, for each player $i \in N$, a preorder $\succcurlyeq_{i}$ over $\mathscr{N}_{i}$, the set of coalitions to which $i$ can belong,

$$
\mathscr{N}_{i}=\{S \subseteq N: S \ni i\} .
$$

The preorder is defined as follows. For all $S, T \in \mathscr{N}_{i}$,

$$
u\left(|S|, \delta_{i}(S)\right) \geq u\left(|T|, \delta_{i}(T)\right) \Leftrightarrow S \succcurlyeq_{i} T .
$$

For each player $i$, the preorder $\succcurlyeq_{i}$ represents her preferences over coalitions, and we refer to a $n$-tuple of preferences as a preference profile. Therefore, an equivalent definition of the coalition formation game is a pair $(N, \succcurlyeq)$, where $N$ is the set of players and $\succcurlyeq$ stands for $\left(\succcurlyeq_{i}\right)_{i \in N}$, a preference profile such that $\succcurlyeq_{i}$ is a weak ordering over $\mathscr{N}_{i}$. The strict preference relation and the indifference relation are respectively denoted $\succ_{i}$ and $\sim_{i}$. Players' preferences are said to be strict if for all $i \in N$, and all $S, T \in \mathscr{N}_{i}$, we either have $S \succ_{i} T$ or $T \succ_{i} S$.

The last fundamental concept we will refer is that of individual rationality. A coalition $S \in \mathscr{N}_{i}$ is individually rational for player $i$ if $S \succcurlyeq_{i}\{i\}$, which means that $i$ 's utility level in $S$ is higher or equal to the utility she would receive if she was alone. Throughout this paper, we assume that each individual is always able to create her own coalition. Thus, it is natural to focus our study on individually rational coalition structures, that is, coalition structures in which all players belong to an individually rational coalition.

\section{Tiebout equilibrium}

The first equilibrium concept we study is that of Tiebout equilibrium. This refers to the case where only individual decisions are allowed, but all 
players are free to enter any existing coalition. We call it an equilibrium with free mobility. This concept has been introduced by Tiebout (1956), who described it as a situation in which agents "vote with their feet." However, the framework proposed by Tiebout (1956) was fairly unclear, and many interpretations arose. Some authors defined the Tiebout equilibrium as an equilibrium where only individual moves are allowed, others interpret Tiebout's (1956) equilibrium concept as a strong Nash like or a core like concept — e.g., Guesnerie and Oddou (1979). We follow in this paper Westhoff's (1977) interpretation, which is an equilibrium with individual moves.

Definition 5 A partition $\mathscr{B}=\left\{S_{1}, \ldots, S_{k}\right\}$ is a Tiebout equilibrium, or a Tiebout-stable coalition structure, if for all $i \in N$, we have,

$$
S(i) \succcurlyeq_{i} T \cup\{i\}, \forall T \in \mathscr{B} \cup\{\varnothing\}
$$

Adding $\{\varnothing\}$ to $\mathscr{B}$ in the definition of the Tiebout equilibrium means that each player is free to create her own coalition. This contrast with other models, such as Westhoff's (1977) or Rose-Ackerman's (1979), who assumed that there is a maximal number of coalitions.

Notice that when the distribution of players is motone, the median voter always lies on the same side of the mean of the extremes. Without loss of generality, we consider now only motone distributions such that the distance between two consecutive players is non-decreasing. Clearly, in this case it holds that $F^{\text {med }}(S) \leq F^{e x t}(S), \forall S \subseteq N$. It turns out that if the decision scheme $F$ does not lie between the median voter and the mean of the extremes, a Tiebout equilibria may not exist.

Proposition 1 If $F(S) \notin\left[F^{\text {med }}(S), F^{e x t}(S)\right]$, then there is a utility function and a strictly monotone distribution of players' positions over $\Omega$ such that no Tiebout equilibrium exists.

This result is new, since usually authors do not tried to characterize which decision schemes ensure the existence of an equilibrium. ${ }^{9}$ Proposition 1 asserts that when the distribution of consumers on $\Omega$ is not uniform, then the mean is not the right concept to be used in order to ensure the existence of a Tiebout equilibrium. Moreover, if we choose the median voter, then the only tie-breaking rule consists of taking the mid-poind between the

\footnotetext{
${ }^{9}$ Barberà and Beviá (1999) also provide a characterization of the decision schemes that ensure the stability of coalitions. However, their model is different from ours as in their model players' utilities do not depend on the size of the coalition.
} 
two median voters. From now on, anytime we shall use the median voter this specific tie-breaking rule will hold.

\subsection{Equilibrium existence}

It turns out that our model makes the task of finding equilibria a difficult one. In fact, as soon as an agent enters a coalition, she induces two effects on her "new partners." First, she has a positive effect, as the size of the coalition increases. But she also has a negative effect due to the shift of the outcome of the decision scheme that her arrival induces. For some players the distance between their position and the outcome of the decision scheme decreases, but for other it increases, and thus the net effect for all players is hard to predict. The next result characterizes the set of Tieboutequilibria for the class of monotone distributions. The regularity showed by these distributions is such that we can somehow master the net effect of the moves performed by players.

To make the point, consider the uniform distribution with $F=F^{e x t}$. Using Lemma 1 in the Appendix, we can restrict ourselves to connected coalitions. Thus, if we consider that the decision scheme $F$ is the mean of the extremes, any player's move to another coalition shifts the decision of her former and her new coalition by an equal amount. ${ }^{10}$ In other words, if $p$ denotes the distance between any two consecutive players in $\Omega$, i.e., $\left|p_{i}-p_{i-1}\right| \equiv p \equiv\left|p_{i}-p_{i+1}\right|, p>0$, then for $i$ entering coalition $S$ we have $F(S \cup\{i\})=F(S) \pm \frac{p}{2}$. Whether one adds $p / 2$ or substracts $p / 2$ from $F(S)$ depends on the relative positions of $i$ and $F(S)$.

Consider now agent $i$ who is at the border of her coalition. That is, consider coalitions,

$$
\left\{p_{i}, p_{i+1}, \ldots, p_{i+k}\right\}, \text { with } k=0, \ldots, n-i,
$$

or coalitions,

$$
\left\{p_{k}, p_{k+1}, \ldots, p_{i}\right\} \text {, with } k=1, \ldots, i \text {. }
$$

Let $\mathscr{S}_{i}$ be the set of coalitions that satisfy equation (4) or (5). We claim that for any coalition $S \in \mathscr{S}_{i}$, its size is sufficient to infer the utility level of agent $i$. Indeed, any player $j$ such that both $S$ and $S \cup\{j\}$ belong to $\mathscr{S}_{i}$ has a positive effect and a negative effect on the utility of player $i$. As we supposed that $u$ is concave with respect to its two arguments, it implies that there is

\footnotetext{
${ }^{10}$ Of course, this only holds if the former coalition was not a one player coalition.
} 
a critical coalitional size $h^{*}$, such that for coalitions in $\mathscr{S}_{i}$ of size larger than $h^{*}$, the shift in $\delta_{i}$ (which is equal to $p / 2$ ), outweighs the effect on the utility due to the increase in the coalition size. Conversely, for coalitions in $\mathscr{S}_{i}$ of size smaller than $h^{*}$, the increase in coalition size outweighs the shift in $\delta_{i}$. Thus, for any player, preferences over coalitions in $\mathscr{S}_{i}$ can be summarized in preferences over coalition sizes, and these preferences are single peaked. Formally, this means that,

$$
\exists h^{*} \in \mathbb{N} \text { such that }|S| \leq|T| \leq h^{*} \Rightarrow T \succcurlyeq_{i} S,
$$

and

$$
h^{*} \leq|T| \leq|S| \Rightarrow T \succcurlyeq_{i} S,
$$

where $h^{*}$ is called the peak. Clearly, if the distribution is strictly monotone, then agents may have two peaks, one for coalition satisfying (4), and one for coalitions satisfying (5). We now state our existence result.

Proposition 2 If the distribution of players' positions on $\Omega$ is monotone and if $F$ is between the mean of the extremes and the median voter, then a Tiebout-equilibrium always exists.

A straightforward consequence of propositions 1 and 2 is the following theorem.

Theorem 1 If the distribution of players' positions on $\Omega$ is monotone, then a Tiebout-equilibrium always exist if and only if $F$ is between the mean of the extremes and the median voter.

In the proof of proposition 2 we construct a coalition structure such that only one kind of move is worthy for players. We then show that if even if such moves occur, then back moves are unworthy. The conclusion rests then on the finiteness of the player set, and hence the number of moves. ${ }^{11}$ However, if we construct a coalition structure in the same way we did in the proof of proposition 2, if a player moves from one coalition to another, we cannot avoid subsequent moves performed by other players in the opposite direction when the distribution of players' positions is not monotone. This claim is an immediate consequence of the following example.

\footnotetext{
${ }^{11}$ A comparable argument is used in Milchtaich and Winter (1998) in their proofs of theorems 1 and 2, which establish the existence of Tiebout equilibrium in their model.
} 
Example 1 Let the set of players be $N=\{a, b, c, d, e, f\}$ with the following positions on the real line, $p_{a}=0, p_{b}=9, p_{c}=19, p_{d}=27, p_{e}=34$, and $p_{f}=40$. Consider the following utility function,

$$
u(|S|, \delta(S))= \begin{cases}|S| & \text { if } \delta(S) \leq 4, \\ |S|-\beta \delta(S) & \text { if } 4<\delta(S)<\frac{19}{2} \\ |S|-10000 \delta(S) & \text { if } \delta(S) \geq \frac{19}{2}\end{cases}
$$

with $\frac{2}{13}<\beta<\frac{2}{11}$.

First, observe that $b$ is closer to $a$ than $c$. Hence, $b$ prefers $\{a b\}$ to $\{b c\}$. Moreover, the distances between $b$ and $d$, and $c$ and $d$ are respectively greater that the distances between $d$ and $f$ and $d$ and $e$. Thus, for any decision scheme between the median voter and the mean of the extremes, $d$ prefers $\{d e f\}$ to $\{b c d\}$. Notice also that $\{a b c\}$ and all coalitions with four players or more are not individually rational. We then obtain the following preference, for any decision scheme between the median voter and the mean of the extremes. ${ }^{12}$

$\{a b\} \succ_{a}\{a\}$,

$\{b c d\} \succ_{b}\{a b\} \succ_{b}\{b c\} \succ_{b}\{b\}$

$\{b c d\} \succ_{c}\{c d\} \succ_{c}\{b c\} \succ_{c}\{c d e\} \succ_{c}\{c\}$

$\{c d e\} \succ_{d}\{d e\} \succ_{d}\{c d\} \succ_{d}\{d e f\} \succ_{d}\{b c d\} \succ_{d}\{d\}$,

$\{$ def $\} \succ_{e}\{e f\} \succ_{e}\{e d\} \succ_{e}\{c d e\} \succ_{e}\{e\}$,

$\{e f\} \succ_{f}\{d e f\} \succ_{f}\{f\}$.

In this example, no Tiebout equilibrium exists. We can observe that as soon as $c$ is alone, then $d$ joins $\{c\}$. But $b$ prefers $\{b c d\}$ to coalitions $\{a b\}$ and $\{b\}$. As soon as $b$ joins $\{c d\}$, then $d$ joins $\{e f\}$, which yields $b$ to leave $\{b c\}$ to join $\{a\}$. To conclude, observe that if $\{c d e\}$ is formed, then $e$ leaves this coalition to join $\{f\}$.

As the example illustrates, such reverse moves can create cycles. This means that a Tiebout equilibrium may not exist when the distribution of players' positions over $\Omega$ is not monotone.

\subsection{On the symmetry and concavity assumptions}

The assumption that players are symmetric is not new in the literature. In fact, this assumption is common to the three models that are the most

\footnotetext{
${ }^{12}$ We do not present coalitions that are not individually rational. For instance, $\{a b c\}$ is not individually rational for $a$ and $c$, but it is for $b$. Hence, $\{a b c\}$ does not appear in $b$ 's preferences.
} 
similar to ours, namely the models of Jehiel and Scotchmer (1997), Alesina and Spolaore (1997), and Milchtaich and Winter (1998). The following example shows that a Tiebout equilibrium may not exist in our model when players have different utility functions.

Example 2 Let $N=\{a, b\}$ be the set of players and $p_{a}=0$, and $p_{b}=4$ be their positions on $\Omega$. If $F$ is the mean of the extremes, then $F(\{a, b\})=2$. Let the utility functions be,

$u_{a}\left(|S|, \delta_{a}(S)\right)=3|S|-\delta_{a}(S)$, $u_{b}\left(|S|, \delta_{b}(S)\right)=3|S|-\delta_{b}(S)^{2}$.

We then get $u_{a}(1,0)=u_{b}(1,0)=3$, and $u_{a}\left(2, \delta_{a}(\{a b\})\right)=4$ and $u_{b}\left(2, \delta_{b}(\{a b\})\right)=2$. Since $\{a b\} \succ_{a}\{a\}$ and $\{b\} \succ_{b}\{a b\}$ no Tiebout equilibrium exists.

However, we advocate that in the horizontal differentiation case this assumption can be easily justified. As we have seen, the horizontal differentiation model can be interpreted as a location choice model. Thus, agents differ in their location in a geographic space, and therefore it seems natural to think that their transportation costs are identical. Thus, utility levels may represent agents' monetary payoffs in the economy.

Like Symmetry, we can also show that the concavity assumption is necessary for the existence of Tiebout equilibria. This is shown by the following example.

Example 3 Consider five players, $a, b, c, d$ and $e$, whose position on the line are $p_{a}=0, p_{b}=2-\varepsilon, p_{c}=3, p_{d}=4$ and $p_{e}=5$. This distribution is monotone, so we know by proposition 2 that a Tiebout equilibrium exists. Consider the following utility function, with $\varepsilon<0.1$.

$$
u_{i}\left(|S|, \delta_{i}(S)\right)= \begin{cases}|S| & \text { if } \delta_{i}(S) \leq \frac{1}{2}+\frac{\varepsilon}{2}, \\ |S|-2.1 \delta_{i}(S) & \text { if } \frac{1}{2}+\frac{\varepsilon}{2}<\delta_{i}(S) \leq \frac{3}{4}, \\ |S|-2.1 & \text { if } \frac{3}{4} \leq \delta_{i}(S) \leq 1, \\ |S|-2.1-\frac{1}{10} \delta_{i}(S) & \text { if } 1<\delta_{i}(S)<\frac{3}{2}+\frac{\varepsilon}{2}, \\ |S|-5-100 \delta_{i}(S) & \text { if } \frac{3}{2}+\frac{\varepsilon}{2} \leq \delta_{i}(S) .\end{cases}
$$

It is easy to show that for any decision scheme lying between the median voter and the mean of the extremes, we get the following preference profile (again, only individualy rational coalitions are represented).

$\{a b c\} \succ_{a}\{a\}$

$\{a b c\} \succ_{b}\{b c\} \succ_{b}\{b c d\} \succ_{b}\{b\}$ 
$\{b c\} \sim_{c}\{c d\} \succ_{c}\{c d e\} \succ_{c}\{a b c\} \succ_{c}\{c\}$,

$\{c d e\} \succ_{d}\{c d\} \sim_{d}\{d e\} \succ_{d}\{b c d\} \succ_{d}\{d\}$,

$\{d e\} \succ_{e}\{c d e\} \succ_{e}\{e\}$.

We leave to the reader to check that there does not exist any Tieboutstable coalition structure.

Hence, concavity with respect to $\delta$ is also necessary to ensure the existence of Tiebout equilibria. ${ }^{13}$

\section{$4 \quad \mathcal{C}$-stable coalition structures}

The second equilibrium concept we use is that of $\mathcal{C}$-stable coalition structures, which was first introduced by Guesnerie and Oddou (1979) for TUgames, and later adapted by Greenberg and Weber (1986) for NTU-games.

As in the Tiebout equilibrium, a player is free to leave her coalition, but now, a player can enter a coalition only if all its members are better off when accepting this new entrant. This somewhat refers to a club, where before a new member joins, its current members proceed to a vote. Here, the vote is by unanimous consent.

Definition 6 A coalition structure $\mathscr{B}$ is blocked by a coalition $S \notin \mathscr{B}$ if,

$$
S \succ_{i} S(\mathscr{B}, i), \quad \forall i \in S \text {. }
$$

To make precise the concept of blocking consider any coalition structure $\mathscr{B}$. If there is a group of players $S$ that is not in $\mathscr{B}$, such that all players in $S$ are stricly better off in $S$ than in their respective coalition in $\mathscr{B}$, then we say that $S$ blocks $\mathscr{B}$. Other authors considered a weaker stability concept. They required that when a coalition blocks all players must be as well off as in their respective coalition, and at least one player must be strictly better off. It turns out that this stability concept may easily prevent $\mathcal{C}$-stable coalition structures to exist. For instance, consider three players, $a, b$ and $c$, uniformly distributed on $\Omega$, and utility functions such that $\{i b\} \succ_{i}\{i\} \succ_{i}\{i b j\}$, with $i \subset\{a b\}$ and $j=\{a b\} \backslash\{i\}$. Clearly, if we use the mean of the extremes, then we have $\{a b\} \sim_{b}\{b c\}$. Thus, the coalition structure $\{\{a b\},\{c\}\}$ is blocked by

\footnotetext{
${ }^{13}$ As the size of the coalition and the distance to the outcome of the decision scheme plays somewhat similar roles, an example in the same vein of example 3 where concavity with respect to the coalition size does not hold and inexistence of Tiebout equilibria can be found.
} 
coalition $\{b c\}$ and the coalition structure $\{\{a\},\{b c\}\}$ is blocked by coalition $\{a b\}$. Hence, there does not exist any $\mathcal{C}$-stable coalition structure.

Given the moves allowed, if a coalition structure is blocked, then it cannot be a candidate for stability. Indeed, under the assumptions of free exit and freedom for any group of players to make a new coalition, if a coalition $S$ blocks a coalition structure $\mathscr{B}$ then members of $S$ will leave their coalition, which will disrupt $\mathscr{B}$. Hence, a coalition structure is $\mathcal{C}$-stable if it not blocked by any coalition. The next definition gives a precise definition of $\mathcal{C}$-stability.

Definition 7 A coalition structure $\mathscr{B}=\left\{S_{1}, \ldots, S_{k}\right\}$ is $\mathcal{C}$-stable if there is no coalition $S \notin \mathscr{B}$ that blocks $\mathscr{B}$.

We now introduce the main result of this section.

Proposition 3 If $F$ is the mean of the extremes then there always exists a $\mathcal{C}$-stable coalition structure.

\subsection{On the symmetry assumption}

Again, the assumption that players have the same utility function plays a crucial role. The next example shows that even if positions are uniformly distributed and $F$ is the mean of the extremes, a $\mathcal{C}$-stable coalition structure may not exist.

Example 4 Consider 8 players, $a, b, c, d, e, f$ and $g$, whose positions on $\Omega$ are $p_{a}=0, p_{b}=1, p_{c}=3, p_{d}=5, p_{e}=7, p_{f}=8.9, p_{g}=10.9$. Utilities are given by,

$$
\begin{aligned}
& u_{a}\left(|S|, \delta_{a}(S)\right)= \begin{cases}|S|-10^{-10} \delta_{a}(S) & \text { if } \delta_{d}(S) \leq 3, \\
|S|-100 \delta_{a}(S) & \text { if } \delta_{d}(S)>3,\end{cases} \\
& u_{b}\left(|S|, \delta_{b}(S)\right)=|S|-10^{-10} \delta_{b}(S), \\
& u_{c}\left(|S|, \delta_{c}(S)\right)= \begin{cases}|S|-\delta_{c}(S) & \text { if } \delta_{d}(S) \leq 1, \\
|S|-100 \delta_{c}(S) & \text { if } \delta_{d}(S)>1,\end{cases} \\
& u_{d}\left(|S|, \delta_{d}(S)\right)= \begin{cases}2|S|-0.9 \delta_{d}(S) & \text { if } \delta_{d}(S) \leq 2, \\
2|S|-\frac{3}{2} \delta_{d}(S) & \text { if } 2<\delta_{d}(S),\end{cases} \\
& u_{e}\left(|S|, \delta_{e}(S)\right)=2|S|-100 \delta_{e}(S),
\end{aligned}
$$




$$
\begin{aligned}
& u_{f}\left(|S|, \delta_{f}(S)\right)=2|S|-10^{-10} \delta_{f}(S), \\
& u_{g}\left(|S|, \delta_{g}(S)\right)=2|S|-10^{-10} \delta_{g}(S) .
\end{aligned}
$$

We then get the following preferences over coalitions (like in example 1 , only the coalitions that are individually rational are presented),

$$
\begin{aligned}
& \{a b c d\} \succ_{a}\{a b\} \succ_{a}\{a\}, \\
& \{a b c d\} \succ_{b}\{b c d\} \succ_{b}\{b f g\} \succ_{a}\{a b\} \succ_{b}\{b d\} \succ_{b}\{b f\} \succ_{b}\{b g\} \succ_{b}\{b\}, \\
& \{a b c d\} \succ_{c}\{b c d\} \succ_{c}\{c\}, \\
& \{d e f\} \sim_{d}\{b c d\} \succ_{d}\{a b c d\} \succ_{d}\{b d\} \sim_{d}\{d f\} \sim_{d}\{d\}, \\
& \{d e f\} \succ_{e}\{e\} \\
& \{b f g\} \succ_{f}\{d e f\} \succ_{f}\{f g\} \succ_{f}\{d f\},\{b f\} \succ_{f}\{f\}, \\
& \{b f g\} \succ_{g}\{f g\} \succ_{g}\{b g\} \succ_{f}\{g\} .
\end{aligned}
$$

Observe that if $e$ is alone, the only possible coalition structures must contain either $\{a b c d\}$ or $\{b c d\}$. Indeed, any coalition structure containing $\{e\}$ and neither $\{a b c d\}$ nor $\{b c d\}$ is blocked by one of these two coalitions. But any coalition structure including these coalitions is blocked by $\{d e f\}$, and any coalition structure with the coalition $\{d e f\}$ is blocked by $\{b f g\}$. In this case, $e$ remains alone, and we then have a cycle. Thus there is no $\mathcal{C}$-stable coalition structure.

\section{$5 \quad$ Unicity and intersection of equilibrium concepts}

Jehiel and Scotchmer (1997) proposed a similar model to the one presented in this paper, but assuming the existence of a continuum of agents. In their paper, Jehiel and Scotchmer supposed that only a positive measure of agents can move. Since their argument was made upon arbitrary infinitesimal moves, an equilibrium with free mobility in their model can almost be considered as a Tiebout equilibrium. One of their results is that the Tiebout equilibrium and the $\mathcal{C}$-stable equilibrium are both unique and do coincide. It turns out that this result does not hold true in our model. Indeed, the coalition structure constructed in the proof of propositions 2 and 3 are not unique. For instance, it can happen that $h^{*}$ divides $|N|$. This would imply that we could construct a coalition structure such that all coalitions have size $h^{*}$, which is obviously Tiebout-stable, and may not necessarily be identical to the coalition structure that we would have obtained with the procedure used in the proof proposition 2. If $h^{*}$ does not divide $|N|$ and the distribution is uniform, one can construct two $\mathcal{C}$-stable coalition structures by using the procedure of the proof of proposition 3 , one starting with player 1 and one starting with player $n$. 
We just showed that the same set of assumptions in our model guarantees the existence of a Tiebout equilibrium and a $\mathcal{C}$-stable coalition structure. However, we can also show that a $\mathcal{C}$-stable coalition structure is not necessarily Tiebout-stable, which contradicts again Jehiel and Scotchmer's results.

Example 5 Let the set of players be $N=\{a, b, c\}$ with the following positions on the real line $p_{a}=0, p_{b}=3$ and $p_{c}=5$, and the following utility function:

$$
u(|S|, \delta(S))= \begin{cases}|S| & \text { if } \delta(S) \leq 1 \\ \left.|S|-0.6 \delta_{(} S\right) & \text { if } 1<\delta(S)<1.1 \\ \left.|S|-0.65 \delta_{(} S\right) & \text { if } 1.1 \leq \delta(S)\end{cases}
$$

For any decision scheme between the mean of the extremes and the median voter, it is easy to see that the only Tiebout stable coalition structure is $\{a b c\}$, and that the only $\mathcal{C}$-stable coalition structure is $\{\{a\},\{b c\}\}$.

The fact that the set of Tiebout equilibria and the set of $\mathcal{C}$-stable coalition structures do not coincide is essentially due to the presence of the decision scheme. As Greenberg and Weber (1993) pointed out, when a player joins a coalition, she does not affect the choice of the coalition, unless there is a new alternative that makes all players better off. The intersection of the set of $\mathcal{C}$-stable and Tiebout stable coalition structures has been called by Greenberg and Weber (1986) the set of strong Tiebout equilibria. In their model, Greenberg and Weber (1986) studied what we defined in this paper as the set of $\mathcal{C}$-stable coalition structures, but without assuming the existence of a decision scheme. In their framework, it turns out that a $\mathcal{C}$ stable coalition structure is also a Tiebout stable coalition structure. The above examples just showed that it is not the case in our model. Combining this observation with Jehiel and Scotchmer's (1997) results, this also shows that a fixed decision scheme has much stronger effects in the finite case than in the infinite case.

\section{Conclusion}

In this paper, we studied the stability of coalition structures when a fixed decision scheme is imposed on coalitions when determining the location of the public good. We showed that in the distribution of players is monotone, then a Tiebout equilibrium always exists if and only if the decision scheme 
lies between the median voter (with the mid-point as the thie-breaking rule) and the Rawlsian decision scheme. This latter proved useful when constructing stable coalition structure. Indeed, under the symmetry assumption, the two extreme players have the same utility level. It can be noticed that these two players are usually the firsts to deviate (particularly in the Tiebout case). Hence, it does not seem surprising that if one wants to ensure the stability of coalition structures, the decision scheme should particularly take care of extreme players. This result contrast with other results recently obtained by Le Breton and Weber (2000). Their model is that of Alesina and Spolaore (1997), which is a model similar to ours, but with a continuum of players and with transferable utilities. They show that there exist compensation schemes, or lump sum transfers, between players located close to the outcome of the decision scheme (the government in their framework) and players close to the borders of coalitions that ensure the existence of $\mathcal{C}$-stable coalition structures. Clearly, our model could be easily written as a TU-game. Provided that with lump sum transfers lemma 1 still holds true, we may conclude using theorem 1 that lump sum transfers can be asymmetric if one uses standard decision schemes such as the median voter. Indeed, theorem 1 says that stability is always ensured if the two extreme players have the same utility level. Hence, we may end up with some cases where one of the extreme players have a positive net transfer, the other one a negative net transfer, and no transfer between intermediate players are necessary.

A last remark is about the contrast between our results and that of previous paper, especially Greenberg and Weber's (1993) paper. Their game has positive externalities, which implied that the set of $\mathcal{C}$-stable coalition structures is also a Tiebout equilibrium. In our game, players' moves may induce negative externalities due to the fixed decision scheme, which can be somehow reinterpreted as congestion effects.

\section{Appendix}

Before proving proposition 1 let us introduce the following result.

Lemma 1 Let $F$ be a decision scheme satisfying Pareto Optimality and Sensitivity. If $\mathscr{B}$ is a Tiebout equilibrium, then $\mathscr{B}$ is connected. 
Proof Let $\mathscr{B}$ be a Tiebout-stable coalition structure, such that there is one coalition, say $S$, that is not connected, i.e., that there exists $T \neq \varnothing$ with $p_{i}<p_{j}<p_{k}$ with $i, k \in S$ and $j \in T$. Because $\mathscr{B}$ is an equilibrium, we have:

$$
u_{i}\left(|S|, \delta_{i}(S)\right)>u_{i}\left(|T|+1, \delta_{i}(T \cup\{i\})\right), \forall i \in S .
$$

Throughout the proof, we shall indifferently use $i$ or $p_{i}$ to denote the same player. We shall show that there exists at least one $i \in S$ such that $u_{i}(|T|+$ $\left.1, \delta_{i}(T \cup\{i\})\right)>u_{i}\left(|S|, \delta_{i}(S)\right)$; or $j \in T$ such that $u_{j}\left(|S|+1, \delta_{j}(S \cup\{j\})\right)>$ $u_{j}\left(|T|, \delta_{j}(T)\right)$ contradicting that $\mathscr{B}$ is an equilibrium - given that $T$ is (partly) surrounded by $S$. Without loss of generality, we shall assume that $F(T) \leq F(S)$. We consider two cases.

(i) $T$ is completely surrounded by $S$. Take $i \in S$ such that $p_{i} \leq p_{k}, \forall k \in$ $T$, and some $j \in T$ such that $p_{j} \leq F(T)$. We obtain the following order: $p_{i}<p_{j}<F(T) \leq F(S)$. Two sub-cases occur.

(a) $F(S \cup j) \leq F(T \cup i)$. We then have $\delta_{j}(S \cup j)<\delta_{j}(T)$, because $F(T \cup i)<F(T)$ and $F(S \cup j)<F(T)$. But $\mathscr{B}$ is a Tiebout equilibrium, we have $u_{j}\left(|T|, \delta_{j}(T)\right) \geq u_{j}\left(|S|+1, \delta_{j}(S \cup j)\right)$. As $\delta_{j}(S \cup j)<\delta_{j}(T)$, we necessarily have $|T|>|S|+1$, otherwise $j$ would deviate to $S$. But notice that $\delta_{i}(T \cup i)<\delta_{i}(S)$. This implies that $u_{i}\left(|T|+1, \delta_{i}(T \cup i)\right)>u_{i}\left(|S|, \delta_{i}(S)\right)$, a contradiction.

(b) $F(S \cup j)>F(T \cup i)$. We suppose that $F(T) \leq F(S \cup j)-$ if the converse inequality would hold, then the argument is the same as in $(a)$. This implies that $|F(T \cup i)-F(S)|>|F(T)-F(S \cup j)|$, which is tantamount to

$$
\left|\delta_{i}(T \cup i)-\delta_{i}(S)\right|>\left|\delta_{j}(T)-\delta_{j}(S \cup j)\right| .
$$

Moreover, as $\mathscr{B}$ is a Tiebout equilibrium, we have: $u_{j}\left(|T|, \delta_{j}(T)\right)>u_{j}(|S|+$ $\left.1, \delta_{j}(S \cup i)\right) \Rightarrow u\left(|T|+1, \delta_{j}(T)\right)>u\left(|S|, \delta_{j}(S \cup j)\right)$ - indices on $u$ in the last inequality are deliberately omitted. It suffices to show that this implies that $u\left(|T|+1, \delta_{i}(T \cup i)\right)>u\left(|S|, \delta_{i}(S)\right)$. But notice that $\delta_{j}(S \cup j)<\delta_{i}(S)$ and (6) imply, together with the concavity of $u$, the desired inequality. Then $i$ moves to $T$, a contradiction.

(ii) $T$ is not completely surrounded by $S$, i.e., $T$ is not connected too. Let $A=\left\{(i, j) \in S \times T:\left|p_{i}-F(S)\right|>\left|p_{j}-F(S)\right|\right\}$. That is, $A$ is the set of couples $(i, j) \in S \times T$ such that $j$ is closer to $F(S)$ than $i$ is. We necessarily have $A \neq \varnothing$, otherwise $S$ would be connected. Let $B=\{(i, j) \in S \times T$ : $\left.\left|p_{i}-F(T)\right|>\left|p_{j}-F(T)\right|\right\}$, the set of couples $(i, j) \in S \times T$ such that $j$ is closer to $F(T)$ than $i$ is. If $A \cap B \neq \varnothing$, then we are back in case (i). Indeed, for $(i, j) \in A \cap B$ we get $\left|\delta_{i}(T \cup i)-\delta_{i}(S)\right|>\left|\delta_{j}(T)-\delta_{j}(S \cup j)\right|$. We are then left 
with the case where there is $(i, j)$ such that $i$ is closer to $F(T)$ than $j$ is and $j$ is closer to $F(S)$ than $i$ is. These two statements respectively imply that $u_{i}\left(|S|, \delta_{i}(S)\right)>u_{i}\left(|T|+1, \delta_{i}(T \cup i)\right) \Leftrightarrow u\left(|S|+1, \delta_{i}(S)\right)>u\left(|T|, \delta_{i}(T \cup i)\right)-$ again, the indices on $u$ are deliberately omitted. This last inequality is tantamount to $u\left(|S|+1, \delta_{j}(S \cup j)\right)>u\left(|T|, \delta_{j}(T)\right)$, which implies that $j$ moves to $S$, a contradiction.

Proof of proposition 1 We first show that if $|N|=2$, then the proposition holds. Let $u$ be such that $u(1,0)=u\left(2, \frac{1}{2}\right)$, i.e., both individuals are indifferent between staying alone and being together. It is straightforward to see that if $F \neq \frac{1}{2}$, no Tiebout equilibrium exists. Without lost of generality, suppose that $F=\frac{1}{2}+\eta$, with $\eta>0$. Then $u_{2}\left(2, \frac{1}{2}-\eta\right)>u_{2}\left(2, \frac{1}{2}\right)$ but $u_{1}\left(2, \frac{1}{2}+\eta\right)<u_{1}\left(2, \frac{1}{2}\right)$. That is, player 2 always makes a coalition with player 1 , but this latter always flees the former (the same argument applies when $F=\frac{1}{2}-\eta$, by reversing player 1 and player 2's roles). Thus the only possibility for an equilibrium when $n=2$ is that $F=\frac{1}{2}$.

We now proceed to the more general case, when $n$ is any positive integer. We must examine two cases, when $F(S)>F^{e x t}(S)$ or $F(S)<F^{\text {med }}(S)$.

Consider the first case, $F(S)>F^{e x t}(S)$, that is, $F(S)=F^{e x t}(S)+$ $\eta$, where $\eta>0 .{ }^{14}$ By lemma 1 , we can restrict to connected coalition structures. Consider a set of players $N=\{1, \ldots, n\}, n \geq 2$, with the following distribution on $\Omega: p_{1}=0, p_{2}=1$ and $\left|p_{h+2}-p_{h+1}\right|=\mid p_{h+1}-$ $p_{h} \mid+\varepsilon, \forall h \in \mathbb{N}$ and $\varepsilon>0$. Thus $p_{s}=s-1+\frac{(s-2)(s-1)}{2} \varepsilon, \forall s \in N$.

Thus, if $N$ is partitioned into two coalitions, $S=\{1, \ldots, s\}$ and $N \backslash S=$ $\{s+1, \ldots, n\}$, and if $F$ is the mean of the extremes,

$$
\begin{aligned}
F(S) & =\frac{1}{2} p_{s}=\frac{s-1}{2}+\frac{(s-2)(s-1)}{2} \varepsilon \\
F(N \backslash S) & =\frac{p_{n}+p_{s+1}}{2}=\frac{n-s-1}{2}+\frac{(n-2)(n-1)-s(s-1)}{2} \varepsilon
\end{aligned}
$$

Thus we deduce,

$$
\begin{aligned}
\delta_{s+1}(S \cup\{s+1\}) & =\frac{s}{2}+\frac{s(s-1)}{4} \varepsilon \\
\delta_{s+1}(N \backslash S) & =\frac{n-s-1}{2}+\frac{(n-1)(n-2)-s(s-1)}{4} \varepsilon
\end{aligned}
$$

\footnotetext{
${ }^{14}$ Using assumption 2 we should have $F(S)=F^{e x t}(S)+\eta(S)$, whith $\eta(S)$ depending on coalition $S$. However, this does not change the argument of the proof.
} 
Let $h^{*}$ be the player such that,

$$
\begin{array}{ll} 
& \delta_{h^{*}}\left(\left\{h^{*}, \ldots, n\right\}\right)<\delta_{h^{*}}\left(\left\{1, \ldots, h^{*}\right\}\right) \\
\text { and } \quad & \delta_{h}(\{h, \ldots, n\})>\delta_{h}(\{1, \ldots, h\}), \forall h<h^{*},
\end{array}
$$

and define $\delta^{*}=\left\{\delta_{h^{*}}\left(\left\{h^{*}, \ldots, n\right\}\right)\right.$. Choose $\varepsilon$ sufficiently small such that $h^{*}=\left\lceil\frac{n}{2}\right\rceil$. Consider the following utility function,

$$
u_{i}\left(|S|, \delta_{i}(S)\right)= \begin{cases}K+s-\beta_{1}\left(\delta_{i}(S)\right) \delta_{i}(S) & \text { if } \delta_{i}(S)<\delta^{*} \\ K+s-\beta_{2}\left(\delta_{i}(S)\right) \delta_{i}(S) & \text { if } \delta_{i}(S) \geq \delta^{*}\end{cases}
$$

where $K>0$ such that any connected coalition is individually rational. Let $\beta_{1}\left(\delta_{i}(\{i\})\right)$ and $\beta_{2}\left(\delta_{i}(N)\right)$ be such that $\{1\} \sim_{1} N$. For $s+1<h^{*}$, let $\delta_{a}(s+1)=\delta_{s+1}(\{1, \ldots, s+1\}), \delta_{b}(s+1)=\delta_{s+1}(\{s+1, \ldots, n\}), \delta_{c}(s+1)=$ $\delta_{n-s}(\{1, \ldots, n-s\})$, and $\delta_{d}(s+1)=\delta_{n-s}(\{n-s, \ldots, n\})$.

Adjust now $\beta_{1}\left(\delta_{s+1}(\{1, \ldots, s+1\})\right)$ and $\beta_{2}\left(\delta_{s+1}(\{s+1, \ldots, n\})\right)$ such that, for $s+1 \leq h^{*}$,

$$
u\left(s+1 ; \frac{\delta_{a}(s+1)+\delta_{d}(s+1)}{2}\right)=u\left(n-s ; \frac{\delta_{b}(s+1)+\delta_{c}(s+1)}{2}\right) .
$$

We posit $\beta_{1}(s+1)=1$.

Claim The utility function defined in eq. (7) satisifies assumptions 3 and 4 and is increasing (resp. decreasing) with respect to the coalition size (resp. distance).

To prove the claim, it suffices to show that $\beta_{2}(s+1)>1$ and that $\beta_{2}(s+1) \geq \beta_{2}(s)$. Indeed, it can be observed that $u$ is decreasing in $\delta$ only if $\beta_{2}(s+1)<\beta_{1}(s+1)$, which implies concavity.

Equation (8) implies that,

$$
s+1-\frac{\delta_{a}(s+1)+\delta_{d} 3(s+1)}{2}=n-s-\beta_{2}(s+1) \frac{\delta_{b}(s+1)+\delta_{c}(s+1)}{2},
$$

which yields,

$$
\beta_{2}(s+1)=\frac{2 n-3 s-2+\gamma(s+1, \varepsilon)}{n-s-1+\gamma^{\prime}(s+1, \varepsilon)}
$$


with,

$$
\begin{aligned}
\gamma(s+1, \varepsilon) & =\frac{(n-2)(n-1)-(n-s-2)(n-s-1)+s(s-1)}{4} \varepsilon \\
\gamma^{\prime}(s+1, \varepsilon) & =\frac{(n-2)(n-1)+(n-s-2)(n-s-1)-s(s-1)}{4} \varepsilon .
\end{aligned}
$$

If we choose $\varepsilon$ sufficiently small, $\beta_{2}(s+1)>1 \forall s<h^{*}$ is tantamount to,

$$
2 n-3 s-2>n-s-1 \Leftrightarrow n-2 s-1>0 .
$$

Clearly, this is trivially satisfied since $s<h^{*}=\left\lceil\frac{n}{2}\right\rceil$. It remains to check that $\beta_{2}(s+1)<\beta_{2}(s)$. That is,

$$
\frac{2 n-3 s-2+\gamma(s+1, \varepsilon)}{n-s-1+\gamma^{\prime}(s+1, \varepsilon)}<\frac{2 n-3 s+1+\gamma(s, \varepsilon)}{n-s+\gamma^{\prime}(s, \varepsilon)}
$$

Again, if we choose $\varepsilon$ sufficiently small, (13) is tantamount to,

$$
\frac{2 n-3 s-2}{n-s-1}<\frac{2 n-3 s+1}{n-s} \Leftrightarrow n-1>0 .
$$

Thus, $u$ is increasing in the coalition size and decreasing in $\delta$, and concave with respect to its two arguments, which completes the proof of the claim.

Because the distribution of players is "increasing", i.e., $\varepsilon>0$, we have $\delta_{a}(s+1)<\delta_{d}(s+1)<\delta^{*}$ and $\delta_{b}(s+1)>\delta_{c}(s+1) \geq \delta^{*}$. Hence, using eq. (8) we have

$$
\begin{aligned}
& u_{s+1}\left(s+1, \delta_{a}\right)>u_{s+1}\left(n+s, \delta_{b}\right), \\
& u_{n-s}\left(n-s, \delta_{c}\right)>u_{n-s}\left(s+1, \delta_{d}\right),
\end{aligned}
$$

Equation (8) is illustrated in figure 1) with

$$
\begin{array}{ll}
u^{*}=u\left(s+1 ; \frac{\delta_{a}(s+1)+\delta_{d}(s+1)}{2}\right)=u\left(n-s ; \frac{\delta_{b}(s+1)+\delta_{c}(s+1)}{2}\right) \\
u_{a}=u\left(s+1 ; \delta_{a}(s+1)\right) & u_{d}=u\left(s+1 ; \delta_{d}(s+1)\right) \\
u_{b}=u\left(n-s ; \delta_{b}(s+1)\right) & u_{c}=u\left(n-s ; \delta_{c}(s+1)\right)
\end{array}
$$

Thus, any coalition structure of the form

$$
\mathscr{B}=\{\{1, \ldots, s\} ;\{s+1, \ldots, n\}\}=\{S ; N \backslash S\}
$$




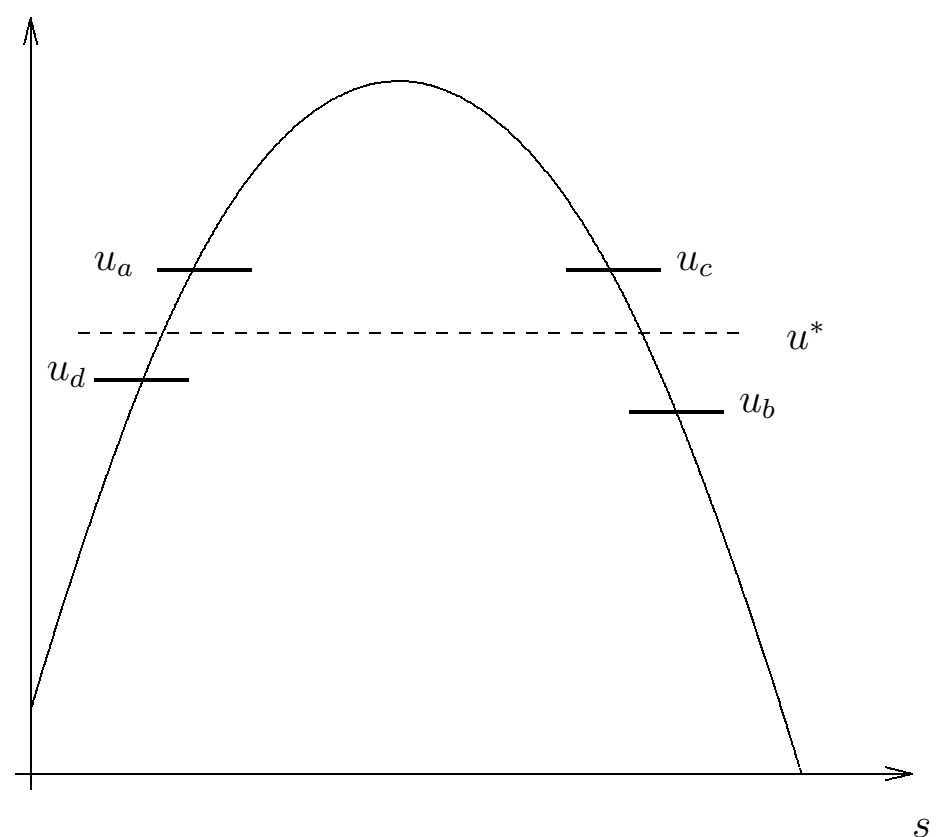

Figure 1: Illustration of eq. (8) and (14).

is unstable unless $S=\{N \backslash\{n\}, N\}$ and $F$ is the mean of the extremes, as $s+1$ prefers to join $\{1, \ldots, s\}$ than staying with $\{s+1, \ldots, n\}$. Moreover, given the shape of $u$, as long as $\mathscr{B}$ has three or more coalitions, $\mathscr{B}$ is unstable as any player at the border of a coalition wants to move to the coalition next to hers, if its size is greater or equal. Indeed, let us suppose that a coalition structure has three or more coalitions. Let us suppose that for some individual $i$ at the border of her coalition, say $S$, we have $\delta_{i}(S)<\delta^{*}$. If $i$ moves to the coalition next to hers, say $T$, we have $\delta_{i}(T \cup\{i\})<\delta^{*}$. For $\varepsilon$ sufficiently small, $i$ will choose the coalition that maximizes the number of members, that is, $i$ chooses $S$ if $s>t+1$ and $T$ otherwise. Conversely, let us suppose that $\delta_{i}(S)>\delta^{*}$. Then, there is a coalition, say $T$, such that $T$ is next to $S$ with $|S|+|T|<|N|$ and an individual at the border of $T$ and next to $S$, say $j$, such that $\delta_{j}(T)<\delta^{*}<\delta_{j}(S \cup\{j\})$. We claim that $u_{j}(S \cup\{j\})>u_{j}(T)$. Let us suppose that $T=\{1, \ldots, t+1\}$ and $S=\{t+2, \ldots, n-k\}$, with $1<k<n-2 t-3$. Without loss of generality, let us suppose that $k=1 .{ }^{15}$ We then have, $u_{j}(S \cup\{j\})>u_{j}(T)$ equivalent

\footnotetext{
${ }^{15}$ We shall see that the difference between $u_{j}(S \cup\{j\})$ and $u_{j}(T)$ does not crucially depend on $k$.
} 
to,

$n-1-s-\hat{\beta}_{2}(t+1)\left(\frac{n-2-t}{2}+\frac{(n-2)(n-3)-t(t-1)}{4} \varepsilon\right)>\frac{t}{2}-\frac{t(t-1)}{4} \varepsilon$,

where $\hat{\beta}_{2}(t+1)$ is $\beta_{2}(t+1)$ in which $n$ is replaced by $(n-1)$ - see equation (10). This is tantamount to,

$$
\begin{aligned}
n-1-\frac{2 n-3 t-4+\hat{\gamma}(t+1, \varepsilon)}{n-t-2+\hat{\gamma}^{\prime}(t+1, \varepsilon)} & \left(\frac{n-t-2}{2}+\frac{(n-2)(n-3)-t(t-1)}{4} \varepsilon\right) \\
& >\frac{3 t}{2}-\frac{t(t-1)}{4} \varepsilon
\end{aligned}
$$

where $\hat{\gamma}$ and $\hat{\gamma}^{\prime}$ are respectively $\gamma$ and $\gamma^{\prime}$ in which $n$ is replace by $n-1-$ see equations (11) and (12). If $\varepsilon$ is sufficiently small, then (16) yields,

$$
\frac{3 t}{2}+1>\frac{3 t}{2} \text {. }
$$

Clearly, the above equation is always satisfied, which proves the claim. Hence, as soon as a player is at the border of two coalitions, say $S$ and $T$ such that $|S|+|T|<|N|$, then she moves to the largest coalition. Thus, the only candidate left for stability is the coalition $N$. Let $F^{*}$ be the value of $F(N)$ if $F$ is the mean of the two extremes. Suppose that $F=F^{*}+\eta$, with $\eta>0$. If $N$ is formed, then 1 is better off if she leaves $N$ and form a coalition by herself, and if $N \backslash\{n\}$ is formed, then $n$ enters $N \backslash\{n\}$. But then 2 joins $1, \ldots$, until $n-1$ joins $\{1, \ldots, n-2\}$, or $n$ joins $\{1, \ldots, n-1\}$. We then have a cycle.

We now proceed to the second case, where $F(S)<F^{\text {med }}(S)$. It is readily verified that by assumption 2 and for $\varepsilon$ small enough, the following inequalities are satisfied

$$
\begin{aligned}
& \delta_{a}(s+1)>\delta_{d}(s+1) \\
& \delta_{b}(s+1)<\delta_{c}(s+1)
\end{aligned}
$$

Hence, the converse of eq. (14) and (15) holds

$$
\begin{aligned}
& u_{s+1}\left(s+1, \delta_{a}\right)<u_{s+1}\left(n+s, \delta_{b}\right), \\
& u_{n-s}\left(n-s, \delta_{c}\right)<u_{n-s}\left(s+1, \delta_{d}\right) .
\end{aligned}
$$

Thus, we obtain the opposite dynamic of the previous case, that is, player $s$ goes from coalition $\{1, \ldots, s\}$ to coalition $\{s+1, \ldots, n\}$. To see that there 
exists a stable coalition structure when $F(S)=F^{\text {med }}(S)$ is easy. Indeed, consider again some player $s+1<h^{*}$. We then have $\delta_{a}(s+1)<\delta_{d}(s+1)$ and $\delta_{b}(s+1)>\delta_{c}(s+1)$. Hence we obtain the same dynamics as in the first case, that is player $s$ goes from coalition $\{s+1, \ldots, n\}$ to coalition $\{1, \ldots, s\}$, except player $n$ who prefers to stay alone than being with $\{1, \ldots, n-1\}$. Thus, any player $k$ has a lower utility than player 1 in coalition $\{1, \ldots, k\}$, which means that the entrance of a player cannot trigger the departure of an opposite player like before.

Proof of proposition 2 We provide the proof for $F=F^{e x t}$. The case for $F=F^{\text {med }}$ is similar. Construct the following coalition structure. If the distribution is monotone, w.l.o.g. say that $\left|p_{2}-p_{1}\right| \geq\left|p_{3}-p_{2}\right|$, then form coalition $S_{1}$ with player 1 and player 2 , add player 3,4 , and so on, until player $i-1$ such that $u_{i}\left(\left|S_{1}\right|+1, \delta_{i}\left(S_{1}\right) \cup\{i\}\right)<u_{i}(1,0)$. Then, form a second coalition, $S_{2}$, with player $i, i+1$, and so on, until player $j-1$ such that $u_{j}\left(\left|S_{2}\right|+1, \delta_{j}\left(S_{2}\right) \cup\{j\}\right)<u_{j}(1,0)$. Repeat this procedure until the last player is reached, yielding the coalition structure $\mathscr{B}=\left\{S_{1}, \ldots, S_{k}\right\}$. Observe that the only players that would wish to move are those in $S_{k-1}$, who move to $S_{k}$. Indeed, by construction, no player in $S_{h+1}$ wants to move to $S_{h}, h=1, \ldots, k-1$, that is, for any $i \in S_{h+1}$, we have $u_{i}\left(\left|S_{h}\right|+1, \delta_{i}\left(S_{h} \cup\{i\}\right)\right)<u_{i}(1,0)$. Because the distribution is monotone, $\delta_{j}\left(S_{h} \cup\{j\}\right) \geq \delta_{i}\left(S_{h} \cup\{i\}\right)$, which implies that $u_{j}\left(\left|S_{h}\right|+1, \delta_{j}\left(S_{h} \cup\{j\}\right)\right)<u_{j}(1,0)$, for any $j \in S_{h-1}$. Consider now $S_{k}$ and $S_{k-1}$. If no player moves from the latter to the former, we are done. Suppose then that there exists $i \in S_{k-1}$ who moves to $S_{k}$. Consequently, some player in $S_{k-2}$ can decide to move to $S_{k-1}$. We claim that $i$ will not decide to move back to $S_{k-1}$. Consider player $i+1$. By construction, we know that $u_{i+1}\left(\left|S_{k-1}\right|+1, \delta_{i+1}\left(S_{k-1} \cup\{i+1\}\right)\right)<u_{i+1}(1,0)$. If $i$ enters $S_{k-1} \cup\{j\} \backslash\{i\}$, then, again because the distribution is monotone, $\delta_{i}\left(S_{k-1} \cup\{j\}\right)<\delta_{i+1}\left(S_{k-1} \cup\{i+1\}\right)$. Thus $u_{i}\left(\left|S_{k-1}\right|+1, \delta_{i}\left(S_{k-1} \cup\{j\}\right)\right)<$ $u_{i}(1,0)$. After $j$ moves, another player in $S_{k-1}, S_{k-2}$ or $S_{k-3}$ may move to $S_{k}, S_{k-1}$ or $S_{k-2}$ respectively, but repeating the argument of before, it can be shown that no player, consequently to such moves, will want to move to a coalition with a lower index.

Proof of proposition 3 Let $N=\{1, \ldots, n\}$ be the set of players. The proof proceeds by construction. 
Step 1 For each player $i$, let $S_{i}^{*} \in \mathscr{S}_{i}$ be the coalition such that $u_{i}\left(\left|S_{i}^{*}\right|, \delta_{i}\left(S_{i}^{*}\right)\right) \geq$ $u_{i}\left(|T|, \delta_{i}(T)\right), \forall T \in \mathscr{S}_{i}$. Observe that $S^{*}$ is connected, and that if $i$ is the player with the lowest (resp. highest) position in $S^{*}$, then $u_{i}\left(\left|S_{i}^{*}\right|, \delta_{i}\left(S_{i}^{*}\right)\right)=$ $u_{j}\left(\left|S_{i}^{*}\right|, \delta_{j}\left(S_{i}^{*}\right)\right)$, where $j$ is the player with the highest (resp. lowest) position in $S^{*}$. Construct now the coalition $S_{1}$ such that $S_{1}=S_{i}^{*}$ for some $i \in N$ and $u_{i}\left(\left|S_{i}^{*}\right|, \delta_{i}\left(S_{i}^{*}\right)\right) \geq u_{j}\left(\left|S_{j}^{*}\right|, \delta_{j}\left(S_{j}^{*}\right)\right)$, for all $j \in N$. We are then left with the set of player $N \backslash S_{i}^{*}$.

Step $k$ Let $N_{k-1}=\left\{S_{1}, \ldots, S_{k-1}\right\}$ be the set of coalitions already formed. Define the set

$$
\hat{\mathscr{S}}_{k, i}=\left\{S \subseteq N \backslash N_{k-1}: i \text { has the lowest or highest position in } S\right\} .
$$

For each $S \in \hat{\mathscr{S}}_{k, i}$, consider the coalitions $T_{1}^{S}, \ldots, T_{l}^{S}$ in $N_{k-1}$ that are surrounded by $S$. That is, for all $1 \leq h \leq l, \exists i, k \in S$ such that $p_{i}<p_{j}<p_{k}$, for all $j \in T_{h}^{S}$. Call a coalition $T_{h}^{S}$ candidate for $S$ if all players in $T_{h}^{S}$ are better off if they join $S: u_{i}\left(\left|T_{h}^{S}\right|+|S|, \delta_{i}\left(T_{h}^{S} \cup S\right)\right)>u_{i}\left(\left|T_{h}^{S}\right|, \delta_{i}\left(T_{h}^{S}\right)\right)$, for all $i \in T_{h}^{S}$. Let $T_{1}^{c, S}, \ldots, T_{l^{\prime}}^{c, S}$ be the collection of candidate coalitions for $S$, and let $\mathscr{T}(S, k-1, i)=\left\{T_{1}^{c, S}\right\} \cup \cdots \cup\left\{T_{l^{\prime}}^{c, S}\right\}$. Define now the set

$$
\mathscr{S}_{k, i}=\left\{S \subseteq \mathscr{T}(S, k-1, i) \cup N \backslash N_{k-1}\right\}
$$

That is, $\mathscr{S}_{k, i}$ is such that if $S$ contains some players in $T_{h}^{c, S}$, then $S$ contains all players in $T_{h}^{c, S}$.

Let $S_{i}^{k, *}$ be the coalition such that $u_{i}\left(\left|S_{i}^{*}\right|, \delta_{i}\left(S_{i}^{*}\right)\right) \geq u_{i}\left(|T|, \delta_{i}(T)\right), \forall T \in$ $\mathscr{S}_{k-1, i}$, Define the coalition $S_{k} \equiv S_{i}^{k-1, *}$, where $i$ is the player such that

$$
u_{i}\left(\left|S_{i}^{k, *}\right|, \delta_{i}\left(S_{i}^{k, *}\right)\right) \geq u_{j}\left(\left|S_{j}^{k, *}\right|, \delta_{j}\left(S_{j}^{k, *}\right)\right), \forall j \in N \backslash N_{k-1} .
$$

Continue this procedure until all players have be assinged to some coalition (it may be that there are one-player coalitions), and call $\mathscr{B}$ the coalition structure obtained. We claim that $\mathscr{B}$ is $\mathcal{C}$-stable.

Clearly, by construction, no collection of coalitions can coalesce to form a coalition, say $T$. Indeed, consider the player, say $i$, with the lowest position in $T$. Observe that $i$ was also the agent with the lowest position in $S(i, \mathscr{B})$, we have $S(i, \mathscr{B})=S_{i}^{k, *}$, where $k$ is the step when $S(i, \mathscr{B})$ was formed. This contradicts the fact that $i$ blocks with $T$, i.e., $u_{i}\left(|T|, \delta_{i}(T)\right)>$ $u_{i}\left(|S(i, \mathscr{B})|, \delta_{i}(S(i, \mathscr{B}))\right)$. Thus, the only possibility is that if some coalition $T$ blocks, it disrupts another coalition $S \in \mathscr{B}$. Two cases occur, whether a disrupted coalition is surrounded by $T$ or at the border of $T$. 
Consider the first case. Let $S$ be the disrupted coalition, with $i, j, k \in S$, $p_{i} \leq p_{j} \leq p_{k}, i, k \in T$ and $j \notin T$. Assume that $i$ and $k$ are respectively the players in $S$ with the lowest and highest position. Because $S$ is surrounded by $T$, there exists some player $h \in T$ such that $p_{k} \leq p_{h}$. Hence, for any player in $l \in S$, we have $F(T \cup\{l\})=F(T)$. Observe that $i$ and $k$ have the same utility level in $S$. Hence, if $i$ blocks with $T$, so does $k$. Let $\Delta_{k}=\delta_{k}(T)-\delta_{k}(S)$. (Define analogously $\Delta_{i}$ and $\Delta_{j}$.) Clearly, we have either $\Delta_{i} \geq \Delta_{j}$ and $\delta_{i}(T) \geq \delta_{j}(T)$ or $\Delta_{k} \geq \Delta_{j}$ and $\delta_{k}(T) \geq \delta_{j}(T)$. Assume that the first pair of inequalities holds. Because $i$ blocks with $T$, we have $u_{i}\left(|S|, \delta_{i}(S)\right)>u_{i}\left(|T|, \delta_{i}(T)\right)$. Hence, by the concavity assumption, it must be also the case that $u_{j}\left(|S|, \delta_{j}(S)\right)>u_{i}\left(|T|+1, \delta_{j}(T)\right)$. In other words, $j$ also blocks with $T$.

Consider now the second case, when a disrupted coalition is at the border of $T$. Let $i \in S$ be the player at the border of $T$. W.l.o.g. suppose that $i$ has the lowest position in $T$. Consider now $j$, such that $p_{i} \leq p_{j}$ and $j$ is at the border of $S$. Suppose first that $i \neq j$. Clearly, $u_{i}\left(|S|, \delta_{i}(S)\right)>u_{j}\left(|S|, \delta_{j}(S)\right)$. But then this contradicts the fact that $j$ is such that $u_{j}\left(\left|S_{j}^{k, *}\right|, \delta_{j}\left(S_{j}^{k, *}\right)\right) \geq$ $u_{i}\left(\left|S_{i}^{k, *}\right|, \delta_{i}\left(S_{i}^{k, *}\right)\right)$, where $k$ is the step when $S$ is formed. If $i=j$, then $S$ being formed instead of $T$ implies that $u_{i}\left(|S|, \delta_{i}(S)\right) \geq u_{i}\left(|T|, \delta_{i}(T)\right)$, a contradiction with the fact that $i$ blocks with $T$, which completes the proof of the claim.

\section{References}

Alesina, A., and E. Spolaore (1997): "On the Number and Size of Nations," Quarterly Journal of Economics, 112, 1027-1056.

Baneruee, S., H. Konishi, and T. Sönmez (1998): "Core in Simple Coalition Formation Game," Social Choice and Welfare, forthcoming.

BarberÀ, S., and C. Beviá (1999): "Self-Selection Consistent Choices," mimeo, Universitat Autónoma de Barcelona.

Barberì, S., And A. Gerber (1999): "On Coalition Formation: Durable Coalition Structures," mimeo, Universitat Autónoma de Barcelona.

Bewley, T. (1981): "A Critique of Tiebout's Theory of Local Public Expenditures," Econometrica, 49, 713-740.

Black, D. (1948): "On the Rationale of Group Decision Making," Journal of Political Economy, 56, 23-34. 
Bogomolnaia, S., And M. Jackson (1998): "The Stability of Hedonic Coalition Structures," mimeo, CalTech.

Demange, G. (1994): "Intermediate Preferences and Stable Coalition Structures," Journal of Mathematical Economics, 23, 45-58.

Drèze, J., And J. Greenberg (1980): "Hedonic Coalitions: Optimality and Stability," Econometrica, 48, 987-1003.

Eppel, D., R. Filimon, and T. Romer (1984): "Equilibrium Amon Local Jurisdictions: Towards and Integrated Treatment of Voting and Residential Choice," Journal of Public Economics, 23, 281-308.

Greenberg, J., and B. Shitovitz (1988): "Consistent Voting Rules for Competitive Local Public Goods Economies," Journal of Economic Theory, 46, 223-236.

Greenberg, J., and S. Weber (1986): "Strong Tiebout Equilibrium under Restricted Preferences Domain," Journal of Economic Theory, 38, $101-117$.

- (1993): "Stable Coalition Structures with a Unidimensional Set of Alternatives," Journal of Economic Theory, 60, 62-82.

Guesnerie, R., and C. Oddou (1979): "On Economic Games that are not Necessarily Superadditive," Economics Letters, 3, 301-306.

Ireland, N. (1987): Product Differentiation and Non-Price Competition. Basic Blackwell, New York.

Jehiel, P., And S. Scotchmer (1997): "Constitutional Rules of Exclusion in Jurisdiction Formation," University of California, Berkeley, working paper $\sharp 231$.

Konishi, H. (1996): "Voting with Ballots and Feet: Existence of Equilibrium in a Local Public Good Economy," Journal of Economic Theory, 68, $480-509$.

Konishi, H., M. Le Breton, and S. Weber (1998): "Equilibrium in a Finite Local Public Good Economy," Journal of Economic Theory, 79, $224-244$.

Le Breton, M., and S. Weber (2000): "The Art of Making Everybody Happy: How to Prevent a Secession," mimeo. 
Milchtaich, I., And E. Winter (1998): "Stability and Segregation in Group Formation," mimeo.

Rose-Ackerman, S. (1979): "Market Models of Local Government: Exit, Voting, and the Land Market," Journal of Urban Economics, 6, 319-337.

Tiebout, C. (1956): "A Pure Theory of Local Public Expenditures," Journal of Political Economy, 65, 319-337.

Westhoff, F. (1977): "Existence of Equilibria in Economies with a Local Public Good," Journal of Economic Theory, 14, 84-112. 